\title{
The Spatial-Temporal Ordering of Amyloid Pathology and Opportunities for PET Imaging
}

\author{
Enrico Fantoni*1, Lyduine Collij*2, Isadora Lopes Alves ${ }^{2}$, Christopher Buckley ${ }^{1}$, and Gill Farrar ${ }^{1}$, on behalf of the \\ AMYPAD consortium \\ ${ }^{I}$ Pharmaceutical Diagnostics, GE Healthcare, Chalfont St. Giles, Buckinghamshire, United Kingdom; and ${ }^{2}$ Department of Radiology \\ and Nuclear Medicine, Amsterdam UMC, Vrije Universiteit Amsterdam, Amsterdam, Netherlands
}

\begin{abstract}
Although clinical routine focuses on dichotomous and visual interpretation of amyloid PET, regional image assessment in research settings may yield additional opportunities. Understanding the regional-temporal evolution of amyloid pathology may enable earlier identification of subjects in the Alzheimer Disease pathologic continuum, as well as a finer-grained assessment of pathology beyond traditional dichotomous measures. This review summarizes current research in the detection of regional amyloid deposition patterns and its potential for staging amyloid pathology. Pathology studies, cross-sectional and longitudinal PET-only studies, and comparative PET and autopsy studies are included. Despite certain differences, cortical deposition generally precedes striatal pathology, and in PETonly studies, medial cortical regions are seen to accumulate amyloid earlier than lateral regions. Based on regional amyloid PET, multiple studies have developed and implemented models for staging amyloid pathology that could improve subject selection into secondary prevention trials and visual assessment in clinical routine.
\end{abstract}

Key Words: amyloid PET; staging; spatial-temporal ordering; pathology

J Nucl Med 2020; 61:166-171

DOI: 10.2967/jnumed.119.235879

$\mathbf{P}$ ET imaging allows the visualization and quantification of amyloid- $\beta(A \beta)$ plaques, a key physiopathologic hallmark for the placement of individuals along the Alzheimer disease (AD) continuum (1). Clinically, brain PET images are visually inspected and reported as a binary negative or positive read after assessment of several regions. Positive scans, indicative of moderate and frequent plaque density, are determined by the presence of elevated signal in at least 1 of 4 or 5 key read regions: frontal lobe, posterior cingulate and precuneus, parietal lobe, lateral temporal lobe, and (specifically for ${ }^{18} \mathrm{~F}$-flutemetamol scans) striatum $(2,3)$. Both research $\left({ }^{11} \mathrm{C}\right.$-Pittsburgh compound-B ( ${ }^{11} \mathrm{C}$-PIB) (4) and commercially approved $\left({ }^{18} \mathrm{~F}\right.$ flutemetamol (5), ${ }^{18} \mathrm{~F}$-florbetaben (6), and ${ }^{18} \mathrm{~F}$-florbetapir (7)) tracers display $85 \%-98 \%$ sensitivity and $87 \%-100 \%$ specificity for $A \beta$ plaques when using the postmortem truth standards of the Consortium to Establish a Registry for Alzheimer's Disease or the National

Received Sep. 6, 2019; revision accepted Dec. 3, 2019.

For correspondence or reprints contact: Gill Farrar, GE Healthcare, Pollards

Wood, Nightingales Lane, Chalfont Saint Giles, HP8 4SP, United Kingdom.

E-mail: gill.farrar@ge.com

${ }^{*}$ Contributed equally to this work.

Published online Dec. 13, 2019.

COPYRIGHT @ 2020 by the Society of Nuclear Medicine and Molecular Imaging.
Institute on Aging-Alzheimer's Association (8-12). Additionally, a focused ${ }^{18} \mathrm{~F}$-flutemetamol PET striatal read accuracy has been demonstrated to detect striatal plaques with $69 \%-87 \%$ sensitivity and $96 \%-100 \%$ specificity (13).

Despite a routine clinical focus on binary negative/positive image interpretation, there is increasing interest in assessment of individual brain regions in the sequential accumulation of amyloid (14). Although dichotomous patient-based PET reading provides the currently approved method to evaluate amyloid deposition $(1,15)$, regional brain measures using quantitative methods may provide valuable information, especially during early phases of accumulation when individuals have few clinical symptoms (1618). Conversely, later in the disease spectrum, regional variations remain more modest, with globally amyloid-positive PET measures in patients with amnestic mild cognitive impairment being comparable in topography and intensity to those in end-of-life AD and non-AD subjects (19).

Taken together, recent reports suggest that regional patterns can be used to identify the earliest signs of amyloid pathology and may indicate potential for conversion to dementia in later stages of the pathologic process. In this context, this article critically synthesizes key research in the field of regional amyloid analysis, with the objective of advancing the overall understanding of amyloid PET's role in the evaluation of subjects in the earlier phases of the $\mathrm{AD}$ spectrum.

\section{REVIEW OF METHODS USED ACROSS THE LITERATURE}

Studies were grouped by methodology. The first group focuses on histopathology at autopsy. Group 2 considers how PET can stage regional amyloid deposition relative to the benchmark ordering method obtained by pathology. The third group assesses the frequency of regional PET amyloid uptake measures in cross-sectional cohorts, whereas the fourth concentrates on longitudinal studies. The studies are summarized in Supplemental Table 1 (supplemental materials are available at http://jnm.snmjournals.org).

\section{Group 1: Postmortem Histology Studies}

Histopathology is considered the gold standard method for amyloid plaque detection (19), with 2 landmark studies. Braak and Braak (20) evaluated the progressive topology and density of amyloid deposits in an analysis of 83 postmortem brains from demented and nondemented elderly patients. Based on both frequency and density of plaques, 3 stages of amyloid deposition were described, starting with deposits in basal portions of the frontal, temporal, and occipital lobes, followed by medium-density deposits throughout cortical association areas and white matter, as well as mild involvement of the hippocampus, and ending with inclusion of the sensorimotor 
cortex and expansion to subcortical structures, particularly striatum. Later, Thal et al. (14) followed a similar methodology but statistically tested for the presence of a constant sequence of regional amyloid deposition, adding strength to their proposed regional sequence compared with Braak albeit disregarding plaque density. In Thal et al., the first phase describes amyloid deposition in the whole neocortex with no mention of within-region differences, in contrast to the relatively more detailed regional assessment in Braak. In turn, 5 phases provide complementary information to Braak's description, focusing on the subsequent spreading of amyloid through subcortical regions.

Although postmortem studies are considered the gold standard, limitations include the modest subject numbers and brain regions evaluated $(14,20)$ and the focus on older end-of-life populations for which other end-stage medical illnesses could further compromise the analysis (8).

\section{Group 2: Studies Comparing Amyloid PET Signal with Histology-Based Regional Thal Staging}

These studies used cohorts with both PET (visual or semiquantitative evaluation) and autopsy data, which are highly informative but generally limited in availability.

Murray et al. (9) showed in a ${ }^{11} \mathrm{C}$-PIB end-of-life cohort of 35 subjects that incremental Thal phasing significantly predicts global SUV ratio (SUVR) signal increases, albeit in a modest population. A follow-up study (21) in 179 end-of-life subjects demonstrated that increases in a global PET measurement scale (centiloid units (22)) are nonlinearly related to Thal phases. Also, global ${ }^{11} \mathrm{C}$-PIB PET detected changes in only Thal phase 2 , missing phase 1 entirely. However, the authors suggested that regional analysis could provide more sensitive estimates of early focal deposits than a global neocortical measure (9).

Similarly, in a 2015 Thal study (23), concordance between visual ${ }^{18}$ F-flutemetamol findings and amyloid phase (Thal phases $0-5$ ) was examined in a dual PET/autopsy 68-subject cohort. PET was visually negative for amyloid in cases that were amyloid phases 1-2, whereas advanced-disease cases of phases 4-5 concurred with a positive scan. In the interim cases, phase 3 , only $33 \%$ were positive, indicating that this phase may represent an inflection point in the progression of amyloid that results in the ability to visually assign positivity to a scan.

Thal et al. additionally demonstrated in an extended cohort of 97 patients that ${ }^{18} \mathrm{~F}$-flutemetamol PET SUVR signal can be streamlined into 0-3 amyloid PET phase estimates. Using signal from a combination of cortical regions and the caudate nucleus, this PET phasing strategy can act as surrogate for Thal phasing between phases 3 and 5 with $97 \%$ accuracy when allowing \pm 1 Thal phase (24).

\section{NOTEWORTHY:}

- The regional-temporal evolution of amyloid pathology may enable the earlier identification of subjects who are in the Alzheimer Disease pathologic continuum.

- Cortical deposition generally precedes striatal pathology, and in PET-only studies, medial cortical regions are seen to accumulate amyloid earlier than lateral regions.

- Models for staging amyloid pathology could improve subject selection into secondary prevention trials and visual assessment in clinical routine.

\section{Group 3: Ranking of Regional Amyloid Signal by PET-Only Studies}

Although limited by the lack of pathologic confirmation, in vivo studies can implement a wide range of strategies to disentangle amyloid deposition patterns and have investigated the regional cortical involvement in more detail than previous postmortem work. To date, most amyloid PET studies have included subjects across the clinical AD spectrum (25-27), and only recent work has studied cognitively unimpaired subjects alone $(28,29)$.

Huang et al. (25) $(n=36)$ used a simple ANOVA between volumes of interest to assess regional ${ }^{18} \mathrm{~F}$-florbetapir PET signal increments across clinical stages. They concluded that amyloid initially accumulates in precuneal, frontal, occipital, and posterior cingulate areas. In a larger dataset $(n=222)$, Villeneuve et al. (26) supported these findings by inferring a sequence of amyloid deposition from statistically significant signals of elevated ${ }^{11} \mathrm{C}-\mathrm{PIB}$ distribution volume ratio among 66 permutations of 22-subject groups of interest with increasing mean ${ }^{11} \mathrm{C}$-PIB distribution volume ratio. With this analytic approach, the authors found an initial deposition of amyloid in medial cortical regions, followed by more lateral regions, finally involving the lateral temporal lobes. Finally, Cho et al. (27) also used cognitively healthy subjects as a reference group to obtain ${ }^{18} \mathrm{~F}$-florbetaben PET Z-transformed SUVRs from 195 subjects across the AD spectrum. The difference in frequency of regional involvement was assessed and then verified with bootstrapping statistics, allowing determination of rank confidence intervals and standard errors. In this work, the precuneus was reported to show early amyloid deposition, whereas the cingulate showed later involvement together with inferior temporal and the insular cortex, somewhat in contrast to previous studies (27).

More recently, studies have focused on amyloid PET signal from cognitively unimpaired subjects, aiming to capture amyloid accumulation, generally more apparent before cognitive impairment $(30,31)$. One key study is by Grothe et al. (28), which in addition to assessing a regional ordering of amyloid deposition was first to create a corresponding staging system based solely on PET imaging. The authors ranked the frequency of regional involvement in amyloid deposition across 179 Alzheimer's Disease Neuroimaging Initiative cognitively unimpaired cases imaged with ${ }^{18} \mathrm{~F}$-florbetapir, essentially translating the traditional methodology from postmortem studies to PET imaging. With this method, 4 stages of amyloid deposition were described, for which early involvement of temporal regions was more pronounced than in previous work (Fig. 1).

Sakr et al., in 2019 (32), further demonstrated that Grothe's 2017 model could be applied to a different cohort of 318 subjective cognitive impairment subjects (33) scanned with ${ }^{18} \mathrm{~F}$-florbetapir PET, with only few subjects deviating from the model. However, when recomputing the model in this subjective cognitive impairment cohort, important discrepancies such as a more frequent involvement of posterior and lateral temporal regions were noted. The authors suggested that age differences between cohorts could explain altered regional deposition patterns due to, for example, late-age comorbidities (32).

Although results from Sakr (32) suggest between-tracer translatability of staging models, this could not be reproduced in work by Lopes Alves et al. (29). In this work, an event-based ordering of regional amyloid deposition was investigated in 3 cohorts $(1,267$ subjects) across the full AD spectrum. Based on a probabilistic approach, a deterministic event-based model (34) learned the temporal ordering of biomarker changes from normal to abnormal in large cross-sectional datasets. Ranking of regions was based on their 


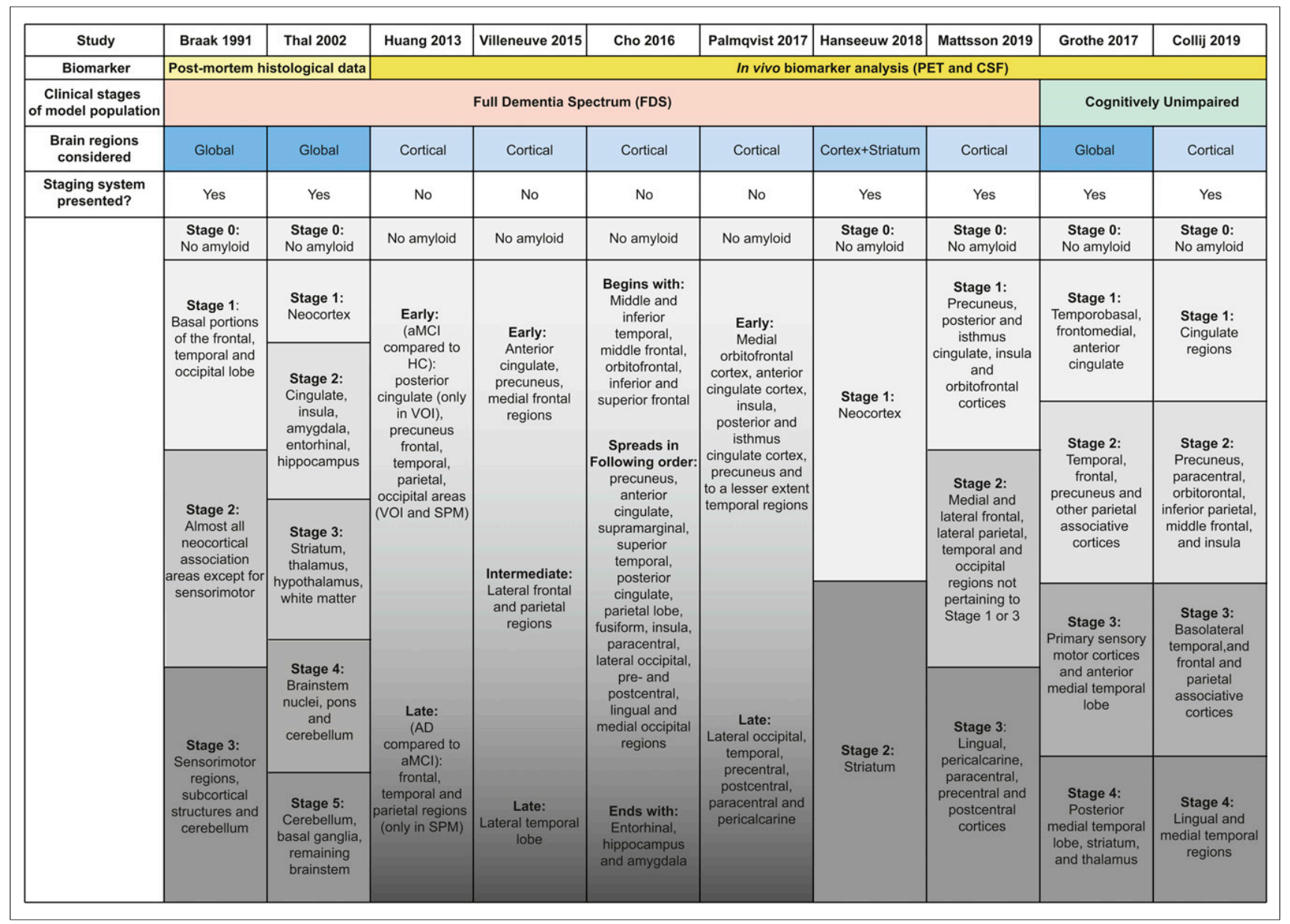

FIGURE 1. General mapping of amyloid deposition order. aMCl = amnestic mild cognitive impairment; CSF = cerebrospinal fluid; $\mathrm{HC}=$ healthy control; SPM = statistical parametric mapping; $\mathrm{VOI}=$ volume of interest.

probability of abnormality, and a populationwide ordering was determined by minimizing cross-subject differences. The 3 radiotracer datasets presented variations in regional probability of accumulation, especially in temporal and frontal regions. In turn, the striatum appeared in later stages for all tracers.

Considering the above, recent work by Collij et al. focused on overcoming the potential lack of cross-cohort and cross-tracer validity for previous staging models (35). Here, a 5-stage model was constructed from PET imaging data from 989 cognitively unimpaired subjects originating from 6 cohorts and scanned with 1 of 4 different PET tracers. The methodology expanded on the earlier work from Grothe et al. (28), with additional tests for abnormality cutoffs and alternative stage definitions. The first stage of deposition started in cingulate regions, followed by precuneus, inferior parietal, paracentral gyrus, lateral orbital, and middle frontal. The insular cortex was involved in stage 2. Then, basal temporal and associative parietal and frontal depositions were present in stage 3 , and finally, other temporal and occipital regions were involved in stage 4 (Fig. 1). These results suggest that by incorporating the intrinsic heterogeneity in populations and available tracers, it is possible to construct a more generalizable staging system, applicable to increasingly common multitracer studies.

Studies in this section describe a range of strategies to understand the regional ordering of amyloid deposition and potentially transform this knowledge into staging systems. Despite differences, different studies were able to translate a temporal pattern of amyloid accumulation into a system for staging amyloid burden. Nonetheless, despite an overall agreement in a broad ordering of amyloid deposition, there is not a complete match in the final staging systems. This indicates that a universal staging system would either have to account for heterogeneity or allow a degree of variability to be accounted for, and further investigation is needed to determine whether the proposed staging models have distinct applications in a clinical setting or to support clinical trials.

\section{Group 4: Studies Based on Longitudinal PET Imaging}

These final studies used longitudinal PET data to derive the sequential ordering of amyloid deposition. These studies are suited to describing the dynamic amyloid spatial-temporal deposition pattern and can effectively account for population differences in $\mathrm{A} \beta$ burden, age-related nonspecific $\mathrm{A} \beta$ accumulation rates, and disease stage heterogeneities.

Hanseeuw et al. (36) based their methods on a priori knowledge from postmortem studies that striatal amyloid deposition is generally preceded by cortical involvement. They hypothesized a higher likelihood of transition of amyloid deposition from cortical to striatal regions than vice versa (14). Longitudinal PET data obtained from ${ }^{18} \mathrm{~F}$-florbetapir and ${ }^{11} \mathrm{C}$-PIB scans from 1,433 patients along the full dementia spectrum were used to assess the hypothesis and define 3 PET stages of amyloidosis: low overall PET signal, high PET signal in cortex only, and high PET signal in both cortex 
and striatum. With the available serial PET measures, individual trajectories advanced successfully through these 3 stages, providing longitudinal support to previous reports showing that the striatum is affected by amyloid deposition only after cortical involvement.

More recently, Palmqvist et al. (37) also assessed the longitudinal patterns in amyloid deposition from PET images, but their analyses were grounded on an independent amyloid biomarker, namely cerebrospinal fluid (CSF) $A \beta_{42}$. The authors hypothesized that early regions of amyloid deposition are those that display increasing accumulation rates (in SUVR/y) when comparing CSF-negative/PET-negative to CSF-positive/PET-negative subjects (38). Conversely, regions where accumulation rates are higher in CSF-positive/PET-positive than in CSF-positive/PET-negative individuals would be those that are affected at a later stage in the disease process. Their results described the cingulate, orbitofrontal, precuneus, and insula as early regions. Central, occipital, temporal, and some limited frontal regions were identified as later regions. Results were then successfully replicated in a separate population from the BioFINDER cohort (39).

Recently, Palmqvist's work has been expanded by Mattsson et al. to construct a longitudinally valid staging model again grounded on CSF $A \beta_{42}$. In addition to the identification of the early and late regions as per Palmqvist's methodology, this work defined an intermediate period by identifying remaining regions with longitudinal accumulation that did not distinguish early or late processes (40). From this replication and extended work, Mattsson et al. were limited to identifying 3 stages of amyloid accumulation. The first stage was defined by the precuneus, the posterior cingulate, the isthmus cingulate, the insula, and the medial and lateral orbitofrontal cortices. The second stage involved the medial and lateral frontal, lateral parietal, temporal, and additional occipital regions, and the final stage described amyloid accumulation in the lingual, pericalcarine, paracentral, precentral, and postcentral cortices (Fig. 1). This model was then applied to 2 cohorts and successfully staged 2,039 PET scans from 741 participants.

\section{DISCUSSION OF APPLIED METHODOLOGIES}

There are important methodologic discrepancies between studies (Supplemental Table 1) such as the presence or absence of histologic postmortem validation, sample size and cohort composition, and whether longitudinal data were present or a temporal ordering was inferred from cross-sectional observations. Some of these methodologic differences may be responsible for the discrepant regional sequences observed. For instance, entorhinal cortical accumulation occurred early in an autopsy study (14), but several PET studies contradicted this (27,35). Cingulate and medial frontal regions showed an early accumulation in most PET studies (26-29,35,37); however, other early regions, such as precuneus, are less robustly reported.

In addition, some more subtle differences inherent to PET imaging may play a role. First, the choice of biomarker may lead to variations in the detection of amyloid plaques $(26,28)$. Stilbenederived amyloid PET radiotracers may be less sensitive for detection of diffuse amyloid than thioflavin-derived tracers, although a head-to-head analysis of tracer uptake would be helpful to confirm this hypothesis $(36,37,41)$. Additionally, a decreased PET sensitivity to amyloid at the extremes of deposition could occur; the first regional deposits with low amyloid concentrations may be missed by global PET imaging $(9,12,23)$, and end-of-life regional deposits are often masked by cerebral atrophy $(2,42)$. PET amyloid tracers also have varying dynamic ranges in terms of the maximum signal strength in positive compared with negative cases (35). These varying ranges could lead to discrepancies when comparing scans from different tracers, hence the value of normalization measures such as the centiloid scale (22). Alternatively, when a tracer harmonization strategy is not implemented, staging models can be made more robust by integrating data from multiple tracers (35).

Furthermore, regional PET signal quantification is subject to methodologic differences across studies even when the same radiotracer is used (Supplemental Table 1). These differences include the inconsistent use of reference regions (26), differences in partialvolume correction techniques $(23,26,32)$, the use of global versus regional quantification (with the latter bearing higher risks of segmentation and coregistration errors) (43), as well as a variable choice of regional and global SUVR cutoffs $(26-28,32)$. Furthermore, the regional detail of staging schemes can vary substantially, with higher levels of detail possibly resulting in increased unstageability rates. Therefore, a balance between abundance of brain regions and complexity of staging scheme is usually necessary to ensure some level of consistency between the studies $(28,32)$. Lack of consistency in the use of PET anatomic atlases and histologic brain sections could also contribute to variability in the overall staging patterns (44).

Lastly, different factors can act as confounders, and only some of the studies include a verification step to ensure that variables do not detract from the robustness of the observations. The presence of a genetic predisposition to $\mathrm{AD}$ (such as the apolipoprotein $\mathrm{E}$ allele $\varepsilon 4$ ) $(36,37,45)$, an imbalanced age distribution (9), cognitive brain reserve (46), brain weight (9), cortical thickness (37), or the presence of widespread diffuse plaques instead of neuritic plaques (41) and comorbidities such as cerebral amyloid angiopathy $(9,32,47)$ can all affect amyloid plaque distribution and the analysis of the PET signal.

The variety of methods used by these studies ultimately provides a broader picture of amyloid accumulation patterns. Generally consistent results across all studies point to the possibility of identifying a high-level ordering of cerebral amyloid deposition, whereas details in regional ordering can be substantially affected by population and tracer differences $(28,32)$ and generalizable staging models may need to allow a degree of heterogeneity to achieve high applicability. Therefore, the use of multiple cohorts and the use of distinct model generation and validation cohorts, biomarkers, and staging methods $(35,40)$ is arguably the most appropriate strategy to derive both a sequence of regional deposition and a staging model with universal validity.

\section{SYNTHESIS OF RESULTS AND OPPORTUNITIES FOR REGIONAL AMYLOID PET}

The diversity of approaches reveals that a universally applicable regional amyloid staging strategy requires consideration of the methodologic discrepancies discussed in this review. Nonetheless, some preliminary all-encompassing agreements about the process of amyloid accumulation can be derived.

\section{Cortical Generally Precedes Striatal Amyloid Accumulation}

Cortical generally precedes striatal accumulation, as is apparent from histologic $(14,20)$ and PET studies that included subcortical regions $(24,28,29,32,35,36)$. Thal et al. (14) justified this order by postulating a sequential amyloid accumulation along neutrally connected areas, with the striatal region receiving inputs from the neocortex (48). Palmqvist (37) and Cho (27) carry this argument a step further, by suggesting a default-mode-network-driven mechanism for the regional accumulation process. In fact, several studies discussed in this review showed that striatal amyloid correlates to worsening cognitive scores and may indicate the potential for transition from preclinical to clinical $\operatorname{AD}(14,28,36)$. However, the region is characterized 
by a predominance of amyloid plaques of diffuse form, to which amyloid tracers are less sensitive than they are to higher-density neuritic plaques $(41,49)$. Yet, the specificity of the thioflavin tracers for detecting varying densities of amyloid plaques in this region is high $(13,50,51)$, and the striatum may therefore have a unique potential to stage patients and assess the risk of disease progression.

\section{In PET Studies, Medial Neocortical Regions Are Seen to Accumulate Amyloid Earlier Than Lateral Regions}

In subjects with moderate amyloid load, medial cortical regions are often more visually intense than lateral regions in amyloid PET scans $(8,52,53)$, despite autopsy evaluation indicating that lateral aspects of temporal, frontal, and parietal lobes are involved earlier in the disease than medial regions $(14,20,47)$. To examine this trend, Smith and Buckley (54) performed a post hoc analysis on a substantial subcohort of subjects from the ${ }^{18} \mathrm{~F}$-flutemetamol autopsy validation cohort $(n=106)(12)$ and examined medial and lateral regions with similar levels of pathology. They showed that medial regions were read positively in over $20 \%$ more cases than lateral regions despite comparable pathology measures.

One possible explanation for these differences is PET signal distortion, derived from the partial-volume overlap effect between regions. The proximity of medial regions to white matter and additional gray matter signal from the contralateral hemisphere could raise a region's relative intensity, adding to true tracer signal. Conversely, in lateral regions, the lack of intensity in juxtaposed regions results in decreased signal due to spill-out into interstitial cranial regions.

As a side effect of this signal distortion, medial regions (easily accessible from a parasagittal plane) can be helpful to visually detect early amyloid deposits with high sensitivity. However, when discussing the true regional amyloid deposition sequence, it is important to consider that the PET signal might represent a heterogeneous distortion of an otherwise uniform tracer distribution. Thus, uncorrected regional averages of quantitative data could influence composite scores and, possibly, the order of staging when early amyloid deposition is examined.

\section{The Placement of Temporal Regions in the Pathologic Ordering Is Remarkably Variable Across Studies}

Early deposition in temporal regions is particularly observed in studies using ${ }^{18} \mathrm{~F}$-florbetapir PET $(25,28,32)$, whereas elsewhere the temporal regions have been described as rather late-accumulating regions $(26,35,37)$, perhaps paralleling the distinct processes of cognitive degeneration and aging as has been suggested by Chételat's group $(55,56)$. From the studies using ${ }^{18} \mathrm{~F}$-florbetapir PET covered here, only Palmqvist (37) and Mattsson (40) do not report temporal regions as among the earliest to display amyloid accumulation. Compared with other ${ }^{18} \mathrm{~F}$-florbetapir studies, the main difference in these studies can be attributed to the use of longitudinal PET imaging to derive the ordering of regional accumulation. In view of Chételat's findings of a distinct pattern between individuals 20$50 \mathrm{y}$ old and elderly subjects, it is possible that temporal regions have an increased signal in midlife and therefore display an elevated PET signal at baseline. This pattern would then be captured when assessing cross-sectional studies of elderly subjects with overall low amyloid PET uptake but would be missed when accounting for longitudinal rates of accumulation starting at $50 \mathrm{y}$ of age or older. However, the reason for this observation's being restricted to ${ }^{18} \mathrm{~F}$-florbetapir scans remains unknown.

\section{CONCLUDING REMARKS}

The quest to determine the most likely temporal order of regional amyloid deposition in AD benefits from studies examining the diverse range of cohorts of ever-increasing size and heterogeneity. The understanding of this progressive accumulation process has considerably evolved since the first regional autopsy analysis in 1991 (20), and methods are becoming more sophisticated, now integrating cohorts using different amyloid biomarkers as well as using diverse techniques to model and validate the order of deposition (Fig. 1).

Broadly speaking, multiple studies indicate that neocortex precedes striatal deposition and that cortical accumulation appears earliest in medial frontal and cingulate regions, then spreading throughout the neocortex and being generally found late in occipital regions. As the pathology progresses, amyloid deposits spread to striatum, hippocampus, and entorhinal regions and then extend further within subcortical regions and into cerebellum. The exact cortical ordering, however, remains diversely described, which perhaps reflects different spatial-temporal deposition patterns inherent to different subpopulations, amyloid detection techniques, and settings (Fig. 1).

Despite residual variability across studies, multiple attempts to create and apply staging systems to assess the extent of amyloid burden with PET imaging have been successful. These efforts indicate there is utility in a relatively broad knowledge of the spatial-temporal development of amyloid plaques. Currently approved amyloid PET imaging methods relate to an overall visual interpretation of brain scans as either positive or negative. However, considering the body of work described here, future image assessment could progress to having an element of regional evaluation using quantitative or semiquantitative PET imaging methods. This could take specific advantage of the high plaque detection sensitivity offered by medial brain regions, as well as of the high specificity toward varying densities of deposited plaques offered by striatal image reads. Regional image reads could be used to assist with identification of subjects for earlier therapeutic intervention and patient staging with a more tailored management approach.

\section{DISCLOSURE}

The project leading to this paper received funding from the Innovative Medicines Initiative 2 Joint Undertaking under grant 115952. This joint undertaking receives support from the European Union's Horizon 2020 research and innovation program and EFPIA. Gill Farrar, Christopher Buckley, and Enrico Fantoni are current or former full-time employees of GE Healthcare. Lyduine Collij and Isadora Lopes Alves are affiliated with the Amsterdam University Medical Center, location VUmc. No other potential conflict of interest relevant to this article was reported.

\section{REFERENCES}

1. Jack CR Jr, Bennett DA, Blennow K, et al. NIA-AA research framework: toward a biological definition of Alzheimer's disease. Alzheimers Dement. 2018;14:535-562.

2. Buckley CJ, Sherwin PF, Smith AP, Wolber J, Weick SM, Brooks DJ. Validation of an electronic image reader training programme for interpretation of $\left[{ }^{18} \mathrm{~F}\right]$ flutemetamol beta-amyloid PET brain images. Nucl Med Commun. 2017;38:234-241.

3. Vizamyl ${ }^{\mathrm{TM}}$ (Flutemetamol F 18 Injection) Electronic Reader Training Program website. https://www.readvizamyl.com/. Accessed December 16, 2019.

4. Klunk WE, Engler H, Nordberg A, et al. Imaging brain amyloid in Alzheimer's disease with Pittsburgh compound-B. Ann Neurol. 2004;55:306-319.

5. Vizamyl: flutemetamol J 18 injection. U.S. Food and Drug Administration website. http://www.accessdata.fda.gov/drugsatfda_docs/label/2017/203137s008lbl.pdf. Revised February 2017. Accessed December 16, 2019 
6. Neuraceq (florbetaben F 18 injection), for intravenous use. U.S. Food and Drug Administration website. http://www.accessdata.fda.gov/drugsatfda_docs/label/2016/ 204677s012lbl.pdf. Revised August 2016. Accessed December 16, 2019.

7. Amyvid (florbetapir F 18 injection) for intravenous use. U.S. Food and Drug Administration website. https://www.accessdata.fda.gov/drugsatfda_docs/label/2013/ 202008s020lbl.pdf. Revised December 2013. Accessed December 16, 2019.

8. Sabri O, Sabbagh MN, Seibyl J, et al. Florbetaben PET imaging to detect amyloid beta plaques in Alzheimer's disease: phase 3 study. Alzheimers Dement. 2015;11:964-974.

9. Murray ME, Lowe VJ, Graff-Radford NR, et al. Clinicopathologic and ${ }^{11} \mathrm{C}$ Pittsburgh compound B implications of Thal amyloid phase across the Alzheimer's disease spectrum. Brain. 2015;138:1370-1381.

10. Clark CM, Pontecorvo MJ, Beach TG, et al. Cerebral PET with florbetapir compared with neuropathology at autopsy for detection of neuritic amyloid-beta plaques: a prospective cohort study. Lancet Neurol. 2012;11:669-678.

11. Curtis C, Gamez JE, Singh U, et al. Phase 3 trial of flutemetamol labeled with radioactive fluorine 18 imaging and neuritic plaque density. JAMA Neurol. 2015;72:287-294.

12. Salloway S, Gamez JE, Singh U, et al. Performance of $\left[{ }^{18} \mathrm{~F}\right]$ flutemetamol amyloid imaging against the neuritic plaque component of CERAD and the current (2012) NIA-AA recommendations for the neuropathologic diagnosis of Alzheimer's disease. Alzheimers Dement (Amst). 2017;9:25-34.

13. Beach TG, Thal DR, Zanette M, Smith A, Buckley C. Detection of striatal amyloid plaques with $\left[{ }^{18} \mathrm{~F}\right]$ flutemetamol: validation with postmortem histopathology. J Alzheimers Dis. 2016;52:863-873.

14. Thal DR, Rub U, Orantes M, Braak H. Phases of A beta-deposition in the human brain and its relevance for the development of AD. Neurology. 2002;58:1791-1800.

15. Dubois B, Hampel H, Feldman HH, et al. Preclinical Alzheimer's disease: definition, natural history, and diagnostic criteria. Alzheimers Dement. 2016;12:292-323.

16. Cohen AD, Mowrey W, Weissfeld LA, et al. Classification of amyloid-positivity in controls: comparison of visual read and quantitative approaches. Neuroimage. 2013;71:207-215.

17. Chételat G, Murray ME. Amyloid PET scan: staging beyond reading? Neurology. 2017;89:2029-2030.

18. Collij LE, Konijnenberg E, Reimand J, et al. Assessing amyloid pathology in cognitively normal subjects using ${ }^{18} \mathrm{~F}$-flutemetamol PET: comparing visual reads and quantitative methods. $J$ Nucl Med. 2019;60:541-547.

19. McKhann GM, Knopman DS, Chertkow H, et al. The diagnosis of dementia due to Alzheimer's disease: recommendations from the National Institute on AgingAlzheimer's Association workgroups on diagnostic guidelines for Alzheimer's disease. Alzheimers Dement. 2011;7:263-269.

20. Braak H, Braak E. Neuropathological stageing of Alzheimer-related changes. Acta Neuropathol (Berl). 1991;82:239-259.

21. La Joie R, Ayakta N, Seeley WW, et al. Multisite study of the relationships between antemortem [ $\left.{ }^{11} \mathrm{C}\right]$ PIB-PET centiloid values and postmortem measures of Alzheimer's disease neuropathology. Alzheimers Dement. 2019;15:205-216.

22. Klunk WE, Koeppe RA, Price JC, et al. The Centiloid Project: standardizing quantitative amyloid plaque estimation by PET. Alzheimers Dement. 2015;11:1-15.e1-4.

23. Thal DR, Beach TG, Zanette M, et al. $\left[{ }^{18} \mathrm{~F}\right]$ flutemetamol amyloid positron emission tomography in preclinical and symptomatic Alzheimer's disease: specific detection of advanced phases of amyloid-beta pathology. Alzheimers Dement. 2015;11:975-985.

24. Thal DR, Beach TG, Zanette M, et al. Estimation of amyloid distribution by $\left[{ }^{18} \mathrm{~F}\right]$ flutemetamol PET predicts the neuropathological phase of amyloid betaprotein deposition. Acta Neuropathol (Berl). 2018;136:557-567.

25. Huang KL, Lin KJ, Hsiao IT, et al. Regional amyloid deposition in amnestic mild cognitive impairment and Alzheimer's disease evaluated by $\left[{ }^{18} \mathrm{~F}\right] \mathrm{AV}-45$ positron emission tomography in Chinese population. PLoS One. 2013;8:e58974.

26. Villeneuve S, Rabinovici GD, Cohn-Sheehy BI, et al. Existing Pittsburgh compound-B positron emission tomography thresholds are too high: statistical and pathological evaluation. Brain. 2015;138:2020-2033.

27. Cho H, Choi JY, Hwang MS, et al. In vivo cortical spreading pattern of tau and amyloid in the Alzheimer disease spectrum. Ann Neurol. 2016;80:247-258.

28. Grothe MJ, Barthel H, Sepulcre J, et al. In vivo staging of regional amyloid deposition. Neurology. 2017;89:2031-2038.

29. Lopes Alves I, Collij L, Heeman F, et al. Event-based modeling of the temporal ordering of regional $\beta$-amyloid deposition in the brain. Alzheimers Dement. 2018;14(suppl):P152.

30. Jack CR Jr, Wiste HJ, Lesnick TG, et al. Brain beta-amyloid load approaches a plateau. Neurology. 2013;80:890-896.

31. Donohue MC, Sperling RA, Petersen R, et al. Association between elevated brain amyloid and subsequent cognitive decline among cognitively normal persons. JAMA. 2017;317:2305-2316.

32. Sakr FA, Grothe MJ, Cavedo E, et al. Applicability of in vivo staging of regional amyloid burden in a cognitively normal cohort with subjective memory complaints: the INSIGHT-preAD study. Alzheimers Res Ther. 2019;11:15.
33. Jessen F, Amariglio RE, van Boxtel M, et al. A conceptual framework for research on subjective cognitive decline in preclinical Alzheimer's disease. Alzheimers Dement. 2014;10:844-852.

34. Venkatraghavan V, Bron EE, Niessen WJ, Klein S. A discriminative event based model for Alzheimer's disease progression modeling. In: Niethammer M, Styner M, Aylward S, et al.., eds. Information Processing in Medical Imaging. New York, NY: Springer; 2017:121-133.

35. Collij L, Heeman F, Salvadó G, et al., on behalf of the AMYPAD consortium. Staging cortical amyloid deposition using PET imaging. Poster presented at: Human Amyloid Imaging Conference; January 18, 2019; Miami, Florida. https:// amypad.eu/wp-content/uploads/2019/01/AMYPAD_Collij_Poster_HAI2019.pdf. Accessed December 16, 2019.

36. Hanseeuw BJ, Betensky RA, Mormino EC, et al. PET staging of amyloidosis using striatum. Alzheimers Dement. 2018;14:1281-1292.

37. Palmqvist S, Scholl M, Strandberg O, et al. Earliest accumulation of betaamyloid occurs within the default-mode network and concurrently affects brain connectivity. Nat Commun. 2017;8:1214.

38. Mattsson N, Insel PS, Donohue M, et al. Independent information from cerebrospinal fluid amyloid-beta and florbetapir imaging in Alzheimer's disease. Brain. 2015;138:772-783.

39. Hansson O, Seibyl J, Stomrud E, et al. CSF biomarkers of Alzheimer's disease concord with amyloid-beta PET and predict clinical progression: a study of fully automated immunoassays in BioFINDER and ADNI cohorts. Alzheimers Dement. 2018;14:1470-1481.

40. Mattsson N, Palmqvist S, Stomrud E, Vogel J, Hansson O. Staging beta-amyloid pathology with amyloid positron emission tomography. JAMA Neurol. July 17, 2019 [Epub ahead of print].

41. Ikonomovic MD, Fantoni ER, Farrar G, Salloway S. Infrequent false positive $\left[{ }^{18} \mathrm{~F}\right]$ flutemetamol PET signal is resolved by combined histological assessment of neuritic and diffuse plaques. Alzheimers Res Ther. 2018;10:60.

42. Seibyl J, Catafau AM, Barthel H, et al. Impact of training method on the robustness of the visual assessment of ${ }^{18} \mathrm{~F}$-florbetaben PET scans: results from a phase3 study. J Nucl Med. 2016;57:900-906.

43. Landau SM, Fero A, Baker SL, et al. Measurement of longitudinal beta-amyloid change with ${ }^{18} \mathrm{~F}$-florbetapir PET and standardized uptake value ratios. $\mathrm{J} \mathrm{Nucl}$ Med. 2015;56:567-574.

44. Schmidt ME, Chiao P, Klein G, et al. The influence of biological and technical factors on quantitative analysis of amyloid PET: points to consider and recommendations for controlling variability in longitudinal data. Alzheimers Dement. 2015;11:1050-1068.

45. Hanseeuw BJ, Jonas V, Jackson J, et al. Association of anxiety with subcortical amyloidosis in cognitively normal older adults. Mol Psychiatry. August 16, 2018 [Epub ahead of print].

46. Bauckneht M, Picco A, Nobili F, Morbelli S. Amyloid positron emission tomography and cognitive reserve. World J Radiol. 2015;7:475-483.

47. Ikonomovic MD, Buckley CJ, Heurling K, et al. Post-mortem histopathology underlying beta-amyloid PET imaging following flutemetamol F 18 injection. Acta Neuropathol Commun. 2016;4:130.

48. Braak H, Braak E, Yilmazer D, de Vos RA, Jansen EN, Bohl J. Pattern of brain destruction in Parkinson's and Alzheimer's diseases. J Neural Transm. 1996;103:455-490.

49. Ikonomovic MD, Klunk WE, Abrahamson EE, et al. Post-mortem correlates of in vivo PiB-PET amyloid imaging in a typical case of Alzheimer's disease. Brain. 2008;131:1630-1645.

50. Klunk WE, Price JC, Mathis CA, et al. Amyloid deposition begins in the striatum of presenilin-1 mutation carriers from two unrelated pedigrees. J Neurosci. 2007;27:6174-6184.

51. Villemagne VL, Ataka S, Mizuno T, et al. High striatal amyloid beta-peptide deposition across different autosomal Alzheimer disease mutation types. Arch Neurol. 2009;66:1537-1544.

52. Vandenberghe R, Van Laere K, Ivanoiu A, et al. ${ }^{18} \mathrm{~F}$-flutemetamol amyloid imaging in Alzheimer disease and mild cognitive impairment: a phase 2 trial. Ann Neurol. 2010;68:319-329.

53. Pontecorvo MJ, Arora AK, Devine M, et al. Quantitation of PET signal as an adjunct to visual interpretation of florbetapir imaging. Eur J Nucl Med Mol Imaging. 2017;44:825-837.

54. Smith A, Buckley C. $\left[{ }^{18} \mathrm{~F}\right]$ flutemetamol PET image representation of Ab pathology; differences between lateral and medial image intensity for equivalent levels of pathology. Presented at: the Human Amyloid Imaging Conference; January 14, 2016; Miami, Florida.

55. Villain N, Chételat G, Grassiot B, et al. Regional dynamics of amyloid-beta deposition in healthy elderly, mild cognitive impairment and Alzheimer's disease: a voxelwise PiB-PET longitudinal study. Brain. 2012;135:2126-2139.

56. Chételat G, Villemagne VL, Villain N, et al. Accelerated cortical atrophy in cognitively normal elderly with high $\beta$-amyloid deposition.Neurology. 2012;78:477-484. 